\title{
AN HI SEARCH FOR M81 GROUP DWARF GALAXIES
}

\author{
W. VAN DRIEL \\ Unité Scientifique Nançay, Observatoire de Paris, France \\ R.C. KRAAN-KORTEWEG \\ Astronomy Department, Universidad de Guanajuato, Mexico \\ B. BINGGELI \\ Astronomical Institute, University of Basel, Switzerland \\ AND \\ W.K. HUCHTMEIER \\ Max-Planck-Institut für Radioastronomie, Bonn, Germany
}

The M81 group has great advantages for dwarf galaxy studies: it has about three times the dwarf content of the Local Group but is at only about a quarter of the Virgo cluster distance. We searched for $\mathrm{HI}$ in 23 optically selected dwarf members and possible members of the M81 group with the Nançay decimetric radio telescope in the velocity range of -529 to $1826 \mathrm{~km} \mathrm{~s}^{-1}$ with considerably better sensitivity $(2-4 \mathrm{mJy}$ rms.) than previous surveys. Half the objects observed are irregular dwarfs, expected to be $\mathrm{H}$ I-rich and detectable at Nançay if at the distance of the M81 group (4 Mpc).

Only three objects (Kar 1N, UGC 4998 and UGC 5658) were detected, and their high radial velocities (between 600 and $1150 \mathrm{~km} \mathrm{~s}^{-1}$ ) show them to lie behind the M81 group. The unexpectedly low detection rate is likely due to confusion with strong foreground (Galactic) as well as local (M81 group) $\mathrm{H}$ I lines: The mean redshift of the M81 group is $\langle V\rangle=95 \mathrm{~km} \mathrm{~s}^{-1}$, while Galactic $\mathrm{H}_{\mathrm{I}}$ emission typically dominates in the range $-150 \leq V \leq 115$ $\mathrm{km} \mathrm{s}^{-1}$, and the local extended $\mathrm{H}$ I envelope in the immediate vicinity of M81 dominates at $-280 \leq V \leq 355 \mathrm{~km} \mathrm{~s}^{-1}$.

$\mathrm{HI}$ emission of low velocity $\mathrm{H}$ I-rich members of the M81 group may thus still remain hidden among strong Galactic and M81 group H I lines optical redshifts are required to determine group memberships (van Driel et al. 1998, $A \& A S, \mathbf{1 2 7}, 1)$. 